\title{
缩醛与吲哚或苯并呋喃的烷基化反应
}

\author{
刘宁宁杨 玲杨蓓王金棒陈雪武钦佩* \\ $\left({ }^{a}\right.$ 北京理工大学化工与环境学院 北京 100081$)$
}

\begin{abstract}
摘要 苯并呋喃衍生物和吲哚衍生物广泛存在于天然产物中，普遍具有生物活性. 缩醛是被频繁使用的前基保护基团. 在 TMSOTf 和三甲基吡啶作用下，芳香基和烷基缩醛都可以直接与苯并呋喃或者吲哚发生烷基化反应; 缩醛的两个烷 氧基被苯并呋喃或者吲哚取代生成双苯并呋喃或双吲哚甲烷衍生物, 与苯并呋喃相比, 吲哚烷基化反应的得率较高. 环状缩醛、含有氨基的缩醛和丙酮缩二甲醇也可以直接发生烷基化反应，反应条件温和. 缩醛的一个烷氧基被吲哚取 代的产物及其被转化为双取代产物的实验结果为反应机理提供了证据.
\end{abstract}

关键词 苯并呋喃; 吲哚; 缩醛; 烷基化

\section{Alkylation of Indole and Benzofuran with Acetals}

\author{
Liu, Ningning Yang, Ling Yang, Bei Wang, Jinbang Chen, Xue Wu, Qinpei* \\ ( ${ }^{a}$ School of Chemical Engineering and Environments, Beijing Institute of Technology, Beijing 100081)
}

\begin{abstract}
Benzofuran derivatives and bisindolylmethane derivatives are widely present in natural products, and have significant biological activity. Acetals were demonstrated to be efficient alkylating agents in the presence of TMSOTf and 2,4,6-collidine. Bisbenzofuranmethane and bisindolylmethane derivatives were easily obtained via direct alkylation of benzofurnan or indole with a series of acetals including amino, cyclic, acetone, aryl and alkyl acetals. The reaction mechanism was proposed.
\end{abstract}

Keywords benzofuran; indole; acetal; alkylation

随着生命科学的发展以及用于调控生理病理的内 源性小分子药物的需求, 吲哚及其衍生物和苯并呋喃类 化合物的研究又成为人们关注的热点之一, 近几年来有 大量的相关文献报道 ${ }^{[1]}$. 双吲哚甲烷衍生物广泛存在于 天然产物中, 而且表现出广谱的抗癌活性. 如图 1 从芦 竹根部提取出来的 3,3'-双吲哚甲烷 (Arundine) 对乳腺 癌 ${ }^{[2]}$ 和前列腺癌 ${ }^{[3]}$ 表现出潜在的抗癌活性; 从海洋细菌 Vibrio parahemolyticus 分离出来的 vibrindole A 对抑郁 以及慢性疲劳综合症具有较好的治疗效果 ${ }^{[4]}$.

已经报道的合成双吲槑甲烷衍生物的方法主要是 羰基化合物和吲哚缩合反应, 所用的催化剂有十二烷基 磺酸 $^{[5]}$ 、氨基磺酸 ${ }^{[6]}$ 、甘油 ${ }^{[7]}$ 、活化硅胶 ${ }^{[8]}$ 、六氟异丙

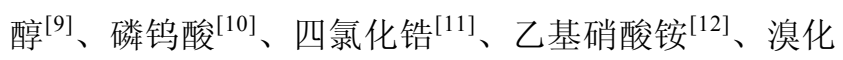

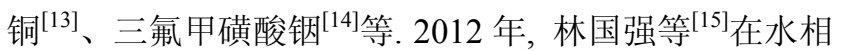
中以三溴化铟为催化剂较高产率的合成了 3 -吲哚取代 的苯酞类化合物. 2008 年, 苏为科等 ${ }^{[16]}$ 采用三氟甲磺酸
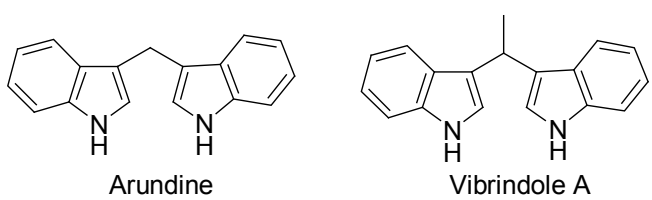

图 1 具有生物活性的吲哚衍生物

Figure 1 Bioactive indole derivatives

铋催化醛和苯并呋喃得到双苯并呋喃甲烷衍生物.

二吲哚甲烷和二苯并呋喃甲烷衍生物大都由醛和 吲哚或者苯并呋喃在酸性条件下缩合制备, 但是, 在多 步合成反应中, 通常需要保护醛基, 缩醛是最常用的保 护醛基的基团; 如果缩醛能够直接参与反应，那就省去 了脱除保护的步骤, 有利于绿色化学合成 ${ }^{[17]}$, 保护环 境. 本文报道三氟甲磺酸三甲基硅脂(TMSOTf)-2,4,6-三 甲基吡啶促进缩醛直接烷基化吲哚以及苯并呋喃的反 应.

\footnotetext{
*E-mail: qpwu@bit.edu.cn

Received June 23, 2014; revised August 10, 2014; published online August 26, 2014.

Project supported by the National Natural Science Foundation of China (No. 21172019).

国家自然科学基金(No. 21172019)资助项目.
} 


\section{1 结果与讨论}

\section{1 反应条件的优化}

以苯甲醛缩二甲醇为反应物, $0{ }^{\circ} \mathrm{C}$ 下, 首先与催化 剂 $\mathrm{Bi}(\mathrm{OTf})_{3}(0.2$ equiv. ) 反应, 约 $30 \mathrm{~min}$ 后缩醛全部转化, 再加入苯并呋喃, 继续反应, $48 \mathrm{~h}$ 后, 仍有缩醛剩余. 为 提高得率, 加入苯并呋喃后, 把反应液的温度升至室温, 4H 后, 缩醛全部转化, 快速色谱柱分离纯化, 核磁谱图 数据表明产物为苯并呋喃取代双烷氧基的 $\mathbf{3 a}$, 得率为 49\%(表 1, Entry 1). 对催化剂的种类和用量进行篮选, 结果表明氯化钴几乎没有催化活性(表 1, Entry 2), $\mathrm{FeCl}_{3}$ 和 TMSOTf 可以催化反应, 产率也是较低(表 1, Entries $3,4)$. 鉴于反应过程 TMSOTf 与缩醛的烷氧基作用生成 TMSOR, 于是增加 TMSOTf 的用量至等量和 2 倍, 但 是, 产率仅略有增加(表 1, Entries 5,6). 向 TMSOTf 的 催化体系中加入 $2,4,6$-三甲基吡啶 ${ }^{[18]}$, 当两者的用量分 别为 $50 \%$ 和 $100 \%$ 时，后者比前者的得率高，当两者用 量都为反应物的 2 倍时, 分离得率为 $81 \%$ (表 1, Entries 7 9). 增加 2,4,6-三甲基吡啶的用量至 3 倍, 多于 TMSOTf 的用量(2.0 eq), 得率为 36\% (表 1, Entry 10). 用三乙胺代替 2,4,6-三甲基吡啶，得率略低(表 1, Entry 11,75\%)，用吡啶(表 1, Entry 12)、三丁基胺(表 1, Entry 13)、 $N, N$-二乙基苯胺(表 1, Entry 14)或者二异丙基乙基 胺(表 1, Entry 15)代替 2,4,6-三甲基吡啶, 得率较低. 分 别用二氯甲烷、乙腈和四氢呋喃作溶剂, 发现在二氯甲 烷中不发生反应，四氢呋喃中反应产率较低，乙腈中反 应得率最高. 因此, 最佳反应条件为: 乙腈为溶剂, 缩 醛先与 TMSOTf (2 equiv.)和 2,4,6-三甲基吡啶(2 equiv.) 在 $0{ }^{\circ} \mathrm{C}$ 反应 $30 \mathrm{~min}$, 再加入苯并呋喃, 反应温度升至室 温.

\section{2 缩醛与苯并呋喃的反应}

在优化的反应条件下, 一系列缩醛与苯并呋喃进行 烷基化反应，结果见表 2 . 由实验结果可见芳香基缩醛 均能与苯并呋喃发生烷基化反应, 以中等到较高产率生 成二苯并呋喃甲烷衍生物(表 2, Entries 1 5), 产率 52\% 93\%. 苯环上含有吸电子基团如硝基的缩醛，反 应产率较高(表 2, Entry 4), 供电子基团, 如甲氧基, 反 应产率较低(表 2, Entry 5). 这可能是因为吸电子基团使 得反应中间体更加稳定. 芳香缩醛间位有硝基时也能得 到中等产率的二苯并呋喃衍生物(表 2, Entry 6), 产率为 $76 \%$. 对于脂肪醛缩醛, 如甲醛缩二甲醇和乙醛缩二乙 醇, 产率较低(表 2, Entries 7,8), 分别为 26\%和 34\%. 这 可能是由于脂肪缩醛类化合物参与反应时所形成的中 间体不稳定所致。
表 1 反应条件的优化

Table 1 Optimization of reaction conditions

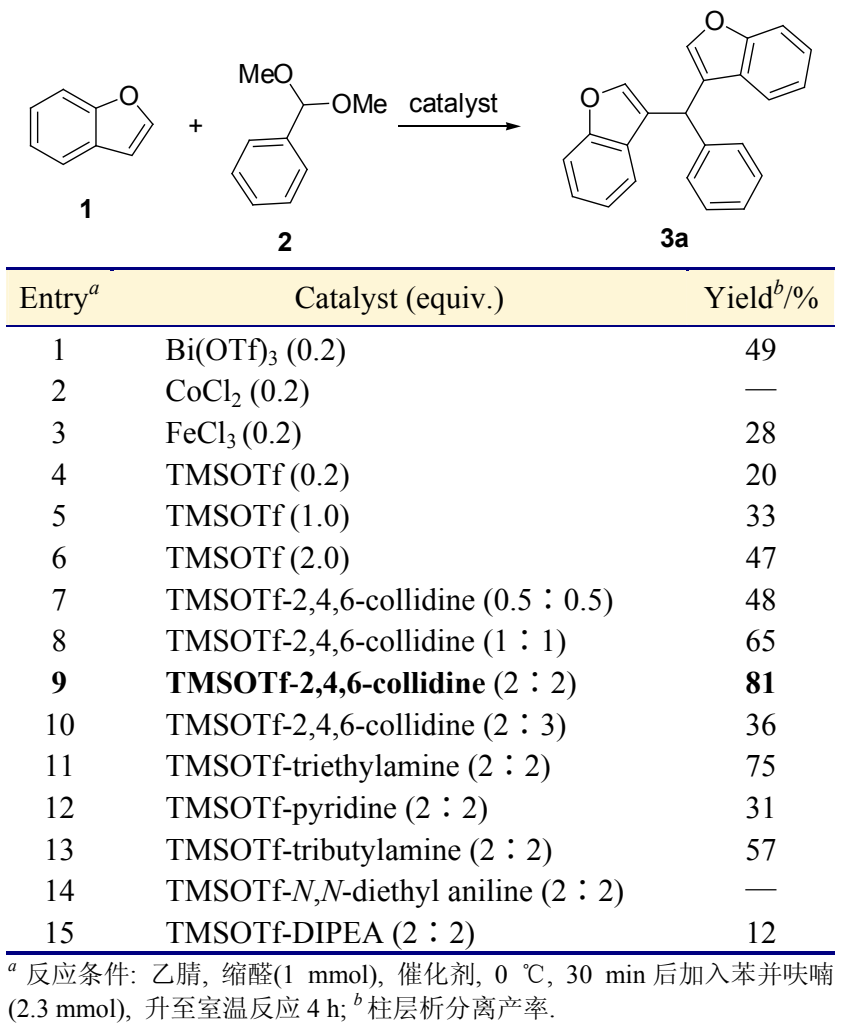

表 2 缩醛和苯并呋喃的烷基化反应

Table 2 Alkylation of benzofuran with acetals

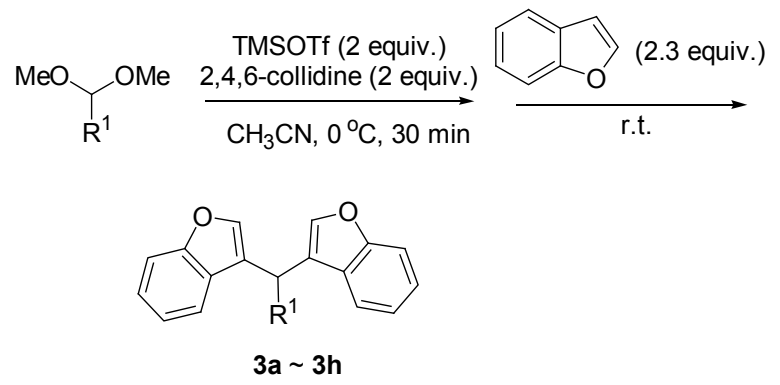

\begin{tabular}{clcc}
\hline Entry $^{a}$ & \multicolumn{1}{c}{$\mathrm{R}^{1}$} & Product & Yield $^{b} \%$ \\
\hline 1 & $\mathrm{C}_{6} \mathrm{H}_{5}$ & $\mathbf{3 a}$ & 81 \\
2 & $p-\mathrm{FC}_{6} \mathrm{H}_{4}$ & $\mathbf{3 b}$ & 73 \\
3 & $p-\mathrm{ClC}_{6} \mathrm{H}_{4}$ & $\mathbf{3 c}$ & 65 \\
4 & $p-\mathrm{NO}_{2} \mathrm{C}_{6} \mathrm{H}_{4}$ & $\mathbf{3 d}$ & 84 \\
5 & $p-\mathrm{CH}_{3} \mathrm{OC}_{6} \mathrm{H}_{4}$ & $\mathbf{3 e}$ & 52 \\
6 & $o-\mathrm{NO}_{2} \mathrm{C}_{6} \mathrm{H}_{4}$ & $\mathbf{3 f}$ & 76 \\
7 & $\mathrm{H}$ & $\mathbf{3 g}$ & 26 \\
8 & $\mathrm{CH}_{3}$ & $\mathbf{3 h}$ & 34 \\
\hline
\end{tabular}

${ }^{a}$ 反应条件: 缩醛 $(1.0 \mathrm{mmol})$, TMSOTf $(2.0 \mathrm{mmol})$ 和 2,4,6-三甲基吡啶 (2.0 $\mathrm{mmol})$, 乙腈, $0{ }^{\circ} \mathrm{C}, 30 \mathrm{~min}$ 后加入苯并呋喃 $(2.3 \mathrm{mmol})$, 升至室温反应 $4 \mathrm{~h}$; ${ }^{b}$ 柱层析分离产率.

\section{3 缩醛与吲哚的反应}

在上述反应条件下, 用吲哚代替苯并呋喃与缩醛进 行烷基化反应，结果列于表 3. 可以看出，与苯并呋喃 
表 3 缩醛与吲哚的烷基化反应 ${ }^{a}$

Table 3 Alkylation of indole with acetals

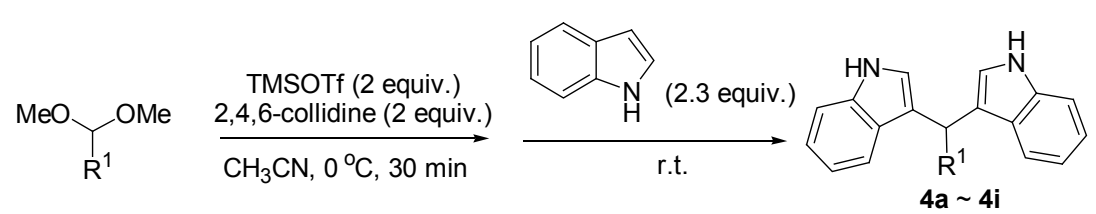

\begin{tabular}{|c|c|c|c|c|}
\hline Entry $^{a}$ & $\mathrm{R}^{1}$ or acetal & Product & m.p. $/{ }^{\circ} \mathrm{C}$ & Yield $^{b} / \%$ \\
\hline 1 & $\mathrm{C}_{6} \mathrm{H}_{5}$ & $4 a$ & $123 \sim 124$ & 96 \\
\hline 2 & $p-\mathrm{FC}_{6} \mathrm{H}_{4}$ & $4 b$ & $117 \sim 119$ & 91 \\
\hline 3 & $p-\mathrm{ClC}_{6} \mathrm{H}_{4}$ & $4 c$ & $78 \sim 79$ & 89 \\
\hline 4 & $p-\mathrm{NO}_{2} \mathrm{C}_{6} \mathrm{H}_{4}$ & $4 d$ & $217 \sim 219$ & 93 \\
\hline 5 & $p-\mathrm{CH}_{3} \mathrm{OC}_{6} \mathrm{H}_{4}$ & $4 e$ & $185 \sim 186$ & 82 \\
\hline 6 & & $4 a$ & $123 \sim 124$ & 93 \\
\hline 7 & & $4 f$ & $137 \sim 138$ & 97 \\
\hline 8 & & $4 \mathrm{~g}$ & $187 \sim 189$ & 50 \\
\hline 9 & $\mathrm{CH}_{3}$ & $4 h$ & $147 \sim 149$ & 68 \\
\hline 10 & & $4 i$ & $109 \sim 110$ & 68 \\
\hline
\end{tabular}

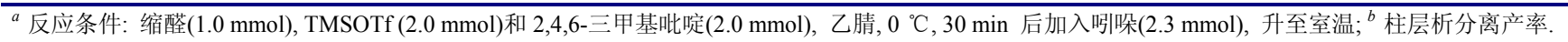

相比，吲哚与缩醛的反应得到双吲哚甲烷衍生物的产率 较高. 同样，含有吸电子基团的芳香族缩醛的烷基化产 物的得率高于含有给电子基团的芳香族缩醛的收率(表 3 , Entries 1 5, 产率为 $82 \% \sim 96 \%$ ). 较为稳定的环状缩 醛的烷基化反应也可以发生, 收率也较高(表 3 Entries 6 8). 含有氨基的苯甲醛缩醛也能够与吲哚发生烷基 化反应, 得到相应的产物 $4 h$ (表 3, Entry 8), 尽管得率较 低, 但是用格氏试剂无法实现这个反应. 另外, 乙醛的 缩醛与吲哚反应得到天然产物二吲哚乙烷(vibrindole A), 产率为 68\%(表 3, Entry 9). 丙酮缩二甲醇也能够发 生烷基化反应, 得到双取代产物(表 3, Entry 10, 产率为 68\%). 烷基缩醛烷基化的得率相对低一些，可能是因为 反应中间体烷基碳正离子的稳定性较低; 但是, 这些反 应的得率远高于烷基化苯并呋喃的得率(表 3 Entries 9, 10 vs 表 2 Entries 7,8).

当丙炔醛二乙基缩醛与吲哚反应时, 我们得到了两 个产物, 分别为单取代 $\mathbf{5 a}$ (得率 $35 \%$ ) 和双取代产物 $\mathbf{5 b}$ (得率 $12 \%$ ), 单取代产物 $\mathbf{5 a}$ 是主要产物, 大约是双取 代产物量的 3 倍. 在类似的反应条件下, 把单取代产物 $5 \mathbf{a}$ 与吲哚反应, 和预想的一样, 5a 顺利地转化为双取代 产物 $5 \mathbf{b}$, 分离纯化得率为 $85 \%$ (图 2). 单取代缩醛烷氧 基产物的获得以及单取代产物转化为双取代产物的反
应结果表明缩醛可以直接发生烷基化反应，也间接地表 明缩醛的烷基化反应经由中间体 I 发生亲电取代反应生 成单取代产物 $\mathbf{I I}^{[18]}$, II 的烷氧基再被取代就生成双取代 烷基化产物 III. 鉴于苠基碳正离子的稳定性高于烷基 碳正离子, 所以, 该反应机理说明了为什么上述芳基缩 醛烷基化反应的产率高于烷基缩醛烷基化反应的产率， 也说明了碱性物质在反应体系中存在的必要性.

\section{2 结论}

在 TMSOTf-2,4,6-collidine 的作用下，芳香基和烷 基缩醛都可以与吲哚或苯并呋喃发生烷基化反应生成 一系列的双吲哚或双苯并呋喃甲烷衍生物，环状缩醛、 含有氨基的缩醛和丙酮缩二甲醇也可以直接发生烷基 化反应，反应条件温和. 缩醛烷基化吲哚的产物得率高 于烷基化苯并呋喃.

\section{3 实验部分}

\section{1 仪器与试剂}

所有试剂均为市售分析纯或化学纯商品, 未进一步 纯化. 熔点用 XT-4A 型显微熔点测定仪，温度未校正； 核磁共振氢谱和碳谱用 Varian mercury-plus 400 型核磁 


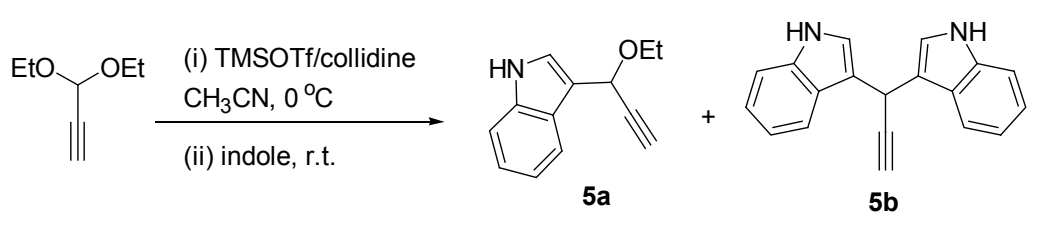

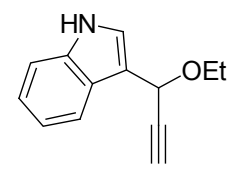

$5 a$

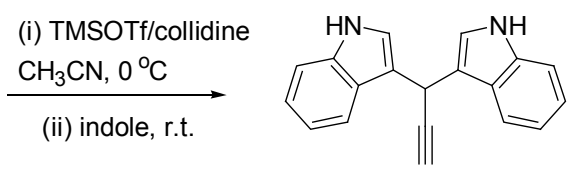

$5 b$

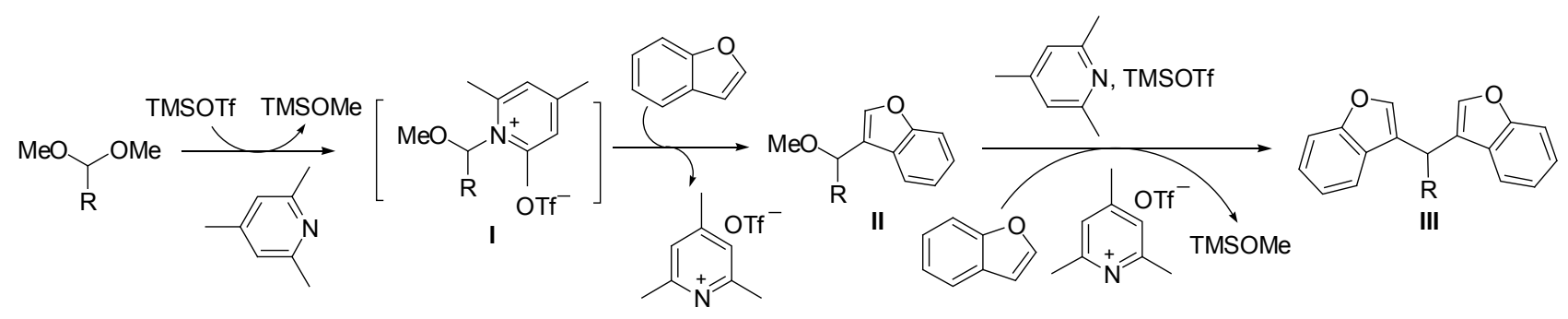

图 2 缩醛烷基化反应机理

Figure 2 Plausible reaction mechanism of alkyltion with acetals

共振仪记录, $\mathrm{DMSO}-d_{6}$ 或 $\mathrm{CDCl}_{3}$ 为溶剂, $\mathrm{TMS}$ 为内标; 乙腈为加入氢化钙回流 $2 \mathrm{~h}$ 后蒸出.

\section{2 实验方法}

缩醛 $(1 \mathrm{mmol}$ )和 2,4,6-三甲基吡啶 $(2 \mathrm{mmol}, 0.3 \mathrm{~mL}$ ) 溶于无水乙腈, 在冰水浴中逐滴加入 TMSOTf $(2 \mathrm{mmol}$, $0.4 \mathrm{~mL}$ ), TLC 跟踪反应, 当缩醛消耗完毕, 加入吲哚或 苯并呋喃 $(2.3 \mathrm{mmol})$, 反应溶液升至室温. 反应完毕后, 加入饱和碳酸氢钠溶液终止反应, 用 $\mathrm{CH}_{2} \mathrm{Cl}_{2}(8 \mathrm{~mL} \times 3)$ 萃取, 合并有机相, 用稀盐酸溶液 $(1 \mathrm{~mol} / \mathrm{L})$ 洗涤有机相 $(5 \mathrm{~mL} \times 2)$, 再用饱和碳酸氢钠溶液洗涤 $(5 \mathrm{~mL} \times 2)$, 合 并有机相, 无水硫酸镁干燥, 过滤, 减压浓缩, 柱层 析.

双(3,3'-苯并呋喃)(苯基)甲烷(3a) ${ }^{[19]}$ : 无色液体, 产 率 $81 \%$. ${ }^{1} \mathrm{H}$ NMR $\left(400 \mathrm{MHz}, \mathrm{CDCl}_{3}\right) \delta: 7.53 \sim 7.61(\mathrm{~m}$, 2H), $7.47(\mathrm{~m}, 2 \mathrm{H}), 7.40 \sim 7.44(\mathrm{~m}, 4 \mathrm{H}), 7.36$ (d, $J=6.5$ $\mathrm{Hz}, 1 \mathrm{H}), 7.22 \sim 7.30(\mathrm{~m}, 4 \mathrm{H}), 6.50(\mathrm{~s}, 2 \mathrm{H}), 5.74(\mathrm{~s}, 1 \mathrm{H})$; ${ }^{13} \mathrm{C}$ NMR $\left(100 \mathrm{MHz}, \mathrm{CDCl}_{3}\right) \delta: 156.89,155.19,138.21$, $128.91,128.76,128.48,127.81,124.14,122.90,120.98$, $111.35,105.32,46.03$.

双(3,3'-苯并呋喃)(4-氟苯基)甲烷(3b) ${ }^{[19]}$ : 无色液体, 产率 73\%. ${ }^{1} \mathrm{H}$ NMR $\left(400 \mathrm{MHz}, \mathrm{CDCl}_{3}\right) \delta: 7.12 \sim 7.43(\mathrm{~m}$, $10 \mathrm{H}), 6.96 \sim 7.05(\mathrm{~m}, 2 \mathrm{H}), 6.39(\mathrm{~s}, 2 \mathrm{H}), 5.60(\mathrm{~s}, 1 \mathrm{H}) ;{ }^{13} \mathrm{C}$ NMR $\left(100 \mathrm{MHz}, \mathrm{CDCl}_{3}\right) \delta: 155.44,154.04,129.25$, 129.12, 127.22, 123.117, 121.835, 119.87, 114.76, 114.55, 110.20, 104.20, 44.11 .

双(3,3'-苯并呋喃)(4-氯苯基)甲烷(3c) $)^{[19]}$ : 无色液体, 产率 65\%. ${ }^{1} \mathrm{H}$ NMR $\left(400 \mathrm{MHz}, \mathrm{CDCl}_{3}\right) \delta: 7.61(\mathrm{~d}, J=7.5$
$\mathrm{Hz}, 2 \mathrm{H}), 7.54(\mathrm{~d}, J=8.5 \mathrm{~Hz}, 2 \mathrm{H}), 7.44 \sim 7.50(\mathrm{~m}, 4 \mathrm{H})$, $7.22 \sim 7.30(\mathrm{~m}, 4 \mathrm{H}), 6.70(\mathrm{~s}, 2 \mathrm{H}), 6.13(\mathrm{~s}, 1 \mathrm{H}) ;{ }^{13} \mathrm{C} \mathrm{NMR}$ $\left(100 \mathrm{MHz}, \mathrm{CDCl}_{3}\right) \delta: 156.61,154.86,137.66,130.81$, $129.32,128.35,125.15,124.76,123.36,121.51,111.54$, $105.53,44.14$.

双(3,3'-苯并呋喃)(4-硝基苯基)甲烷(3d ${ }^{[19]}$ : 无色液 体, 产率 84\%. ${ }^{1} \mathrm{H}$ NMR (400 MHz, $\left.\mathrm{CDCl}_{3}\right) \delta: 8.28(\mathrm{~d}, J=$ $8.8 \mathrm{~Hz}, 2 \mathrm{H}), 7.73(\mathrm{~d}, J=8.8 \mathrm{~Hz}, 2 \mathrm{H}), 7.62 \sim 7.67(\mathrm{~m}, 2 \mathrm{H})$, $7.55 \sim 7.59(\mathrm{~m}, 4 \mathrm{H}), 7.25 \sim 7.30(\mathrm{~m}, 4 \mathrm{H}), 6.36(\mathrm{~s}, 1 \mathrm{H}),{ }^{13} \mathrm{C}$ NMR $\left(100 \mathrm{MHz}, \mathrm{CDCl}_{3}\right) \delta: 155.74,154.96,147.45$, $146.06,130.32,128.30,124.94,124.55,123.59,121.73$, $111.61,106.0,44.43$.

1,1,1-双(3,3'-苯并呋喃)(4-甲氧基苯基)甲烷(3e $)^{[19] \text { : }}$ 无色液体, 产率 $52 \% .{ }^{1} \mathrm{H}$ NMR $\left(400 \mathrm{~Hz}, \mathrm{DMSO}-d_{6}\right) \delta$ : $7.60(\mathrm{~d}, J=7.0 \mathrm{~Hz}, 2 \mathrm{H}), 7.53(\mathrm{~d}, J=8.0 \mathrm{~Hz}, 2 \mathrm{H}), 7.35 \sim$ $7.39(\mathrm{~m}, 2 \mathrm{H}), 7.20 \sim 7.28(\mathrm{~m}, 4 \mathrm{H}), 6.97(\mathrm{~d}, J=8.5 \mathrm{~Hz}$, 2H), $6.65(\mathrm{~s}, 2 \mathrm{H}), 5.98(\mathrm{~s}, 1 \mathrm{H}), 3.75(\mathrm{~s}, 3 \mathrm{H}) ;{ }^{13} \mathrm{C} \mathrm{NMR}$ $\left(400 \mathrm{~Hz}, \mathrm{DMSO}-d_{6}\right) \delta: 159.08,157.52,154.80,130.57$, $130.03,128.43,124.59,123.39,121.53,114.63,111.48$, 105.09, 55.58, 44.15.

双(3,3'-苯并呋喃)(3-硝基苯基)甲烷(3f ${ }^{[19]}$ : 无色油 状物, 产率 76\%. ${ }^{1} \mathrm{H}$ NMR $\left(400 \mathrm{MHz}, \mathrm{CDCl}_{3}\right) \delta: 8.32(\mathrm{t}$, $J=2.0 \mathrm{~Hz}, 1 \mathrm{H}), 8.25 \sim 8.18(\mathrm{~m}, 1 \mathrm{H}), 7.76(\mathrm{t}, J=10.0 \mathrm{~Hz}$, $1 \mathrm{H}), 7.61 \sim 7.53(\mathrm{~m}, 3 \mathrm{H}), 7.49 \sim 7.45(\mathrm{~m}, 2 \mathrm{H}), 7.35 \sim 7.22$ (m, 5H), 6.59 (s, 2H), $5.85(\mathrm{~s}, 1 \mathrm{H}) ;{ }^{13} \mathrm{C}$ NMR $(100 \mathrm{MHz}$, $\left.\mathrm{CDCl}_{3}\right) \delta: 155.29,155.10,148.74,140.40,134.90,129.90$, $128.19,124.64,123.87,123.22,122.99,121.23,111.44$, 
$105.88,45.64$.

双 (3,3'-苯并㫙喃) 甲烷 $(\mathbf{3 g})^{[19]}$ : 无色液体，产率 26\%. ${ }^{1} \mathrm{H}$ NMR (400 MHz, $\left.\mathrm{CDCl}_{3}\right) \delta: 7.55 \sim 7.51(\mathrm{~m}, 2 \mathrm{H})$, $7.47(\mathrm{~d}, J=8.1 \mathrm{~Hz}, 2 \mathrm{H}), 7.30 \sim 7.20(\mathrm{~m}, 4 \mathrm{H}), 6.67 \sim 6.56$ $(\mathrm{m}, 2 \mathrm{H}), 4.31(\mathrm{~s}, 2 \mathrm{H}) ;{ }^{13} \mathrm{C} \mathrm{NMR}\left(100 \mathrm{MHz}, \mathrm{CDCl}_{3}\right) \delta$ : $149.74,148.55,123.44,118.60,117.53,115.45,105.83$, $98.89,23.22$.

双( $3,3^{\prime}$-苯并呋喃)甲基甲烷(3h): 无色液体，产率 34\%. ${ }^{1} \mathrm{H} \mathrm{NMR}\left(400 \mathrm{MHz}, \mathrm{CDCl}_{3}\right) \delta: 7.58(\mathrm{~d}, J=1.6 \mathrm{~Hz}$, $2 \mathrm{H}), 7.51(\mathrm{~d}, J=6.4 \mathrm{~Hz}, 2 \mathrm{H}), 7.18 \sim 7.31(\mathrm{~m}, 4 \mathrm{H}), 6.78(\mathrm{~s}$, $2 \mathrm{H}), 4.64 \sim 4.66(\mathrm{~m}, 1 \mathrm{H}), 1.74 \sim 1.78(\mathrm{~m}, 3 \mathrm{H}) ;{ }^{13} \mathrm{C} \mathrm{NMR}$ $\left(100 \mathrm{MHz}, \mathrm{CDCl}_{3}\right) \delta: 159.08,154.56,128.60,124.37$, 123.30, 121.33, 111.41, 103.20, 33.47, 17.79. HRMS (ESI) calcd for $\mathrm{C}_{18} \mathrm{H}_{14} \mathrm{O}_{2}(\mathrm{M}+\mathrm{H})^{+}$263.1067, found 263.1062.

双(3,3'-1H-吲哚)(苯基)甲烷(4a): 白色固体，258 $\mathrm{mg}$, 产率 96\%. m.p. 123 124 ${ }^{\circ} \mathrm{C}$ (lit. ${ }^{[20]} 124 \sim 125{ }^{\circ} \mathrm{C}$ ); $R_{\mathrm{f}}=0.55$ [ $V($ 乙酸乙酯 $): V($ 石油醚 $\left.)=3: 7\right]$. ${ }^{1} \mathrm{H} \mathrm{NMR}$ $\left(400 \mathrm{MHz}, \mathrm{CDCl}_{3}\right) \delta: 7.73(\mathrm{~s}, 2 \mathrm{H}), 7.30(\mathrm{~d}, J=7.9 \mathrm{~Hz}$, $2 \mathrm{H}), 7.28 \sim 7.22(\mathrm{~m}, 4 \mathrm{H}), 7.21 \sim 7.15(\mathrm{~m}, 2 \mathrm{H}), 7.12(\mathrm{dd}$, $J=6.0,3.8 \mathrm{~Hz}, 1 \mathrm{H}), 7.08(\mathrm{t}, J=7.6 \mathrm{~Hz}, 2 \mathrm{H}), 6.92(\mathrm{t}, J=$ $7.5 \mathrm{~Hz}, 2 \mathrm{H}), 6.52(\mathrm{~d}, J=2.1 \mathrm{~Hz}, 2 \mathrm{H}), 5.79(\mathrm{~s}, 1 \mathrm{H}) ;{ }^{13} \mathrm{C}$ NMR $\left(100 \mathrm{MHz}, \mathrm{CDCl}_{3}\right) \delta: 142.97,135.65,127.70$, 127.19 , 126.06, 125.11, 122.58, 120.90, 118.92, 118.69, $118.21,110.00,39.18$.

双 (3,3'-1H-吲哚)(4-氟苯基)甲烷(4b)：粉色固体, $308 \mathrm{mg}$, 产率 91\%. m.p. $117 \sim 119{ }^{\circ} \mathrm{C}$ (lit. ${ }^{[21]} 118 \sim 120$ $\left.{ }^{\circ} \mathrm{C}\right) ; R_{\mathrm{f}}=0.67$ [ $V($ 乙酸乙酯 $): V($ 石油醚 $\left.)=3: 7\right] .{ }^{1} \mathrm{H}$ NMR (400 MHz, $\left.\mathrm{CDCl}_{3}\right) \delta: 7.84(\mathrm{~s}, 2 \mathrm{H}), 7.29 \sim 7.36(\mathrm{~m}$, 4H), $7.24 \sim 7.17(\mathrm{~m}, 3 \mathrm{H}), 7.10(\mathrm{t}, J=7.4 \mathrm{~Hz}, 2 \mathrm{H}), 6.91(\mathrm{t}$, $J=5.2 \mathrm{~Hz}, 4 \mathrm{H}), 6.56(\mathrm{~d}, J=1.8 \mathrm{~Hz}, 2 \mathrm{H}), 5.79(\mathrm{~s}, 1 \mathrm{H}) ;{ }^{13} \mathrm{C}$ NMR $\left(100 \mathrm{MHz}, \mathrm{CDCl}_{3}\right) \delta: 135.68,129.09,129.01$, $125.90,122.53,121.02,118.84,118.57,118.29,114.04$, $113.83,110.06,38.45$.

双 (3,3'-1H-吲哚)(4-氯苯基)甲烷(4c)：粉色固体， $316 \mathrm{mg}$, 产率 89\%. m.p. $78 \sim 79{ }^{\circ} \mathrm{C}$ (lit. ${ }^{[20]} 76 \sim 78{ }^{\circ} \mathrm{C}$ ); $R_{\mathrm{f}}=0.62[V($ 乙酸乙酯 $): V($ 石油醚 $)=3: 7]$. ${ }^{1} \mathrm{H} \mathrm{NMR}$ $\left(400 \mathrm{MHz}, \mathrm{CDCl}_{3}\right) \delta: 7.87(\mathrm{~s}, 2 \mathrm{H}), 7.34(\mathrm{t}, J=7.4 \mathrm{~Hz}, 4 \mathrm{H})$, $7.27 \sim 7.21(\mathrm{~m}, 4 \mathrm{H}), 7.17(\mathrm{t}, J=7.6 \mathrm{~Hz}, 2 \mathrm{H}), 7.01(\mathrm{t}, J=$ $7.6 \mathrm{~Hz}, 2 \mathrm{H}), 6.60(\mathrm{~d}, J=2.3 \mathrm{~Hz}, 2 \mathrm{H}), 5.84(\mathrm{~s}, 1 \mathrm{H}) ;{ }^{13} \mathrm{C}$ NMR (100 MHz, $\left.\mathrm{CDCl}_{3}\right) \delta: 141.52,135.65,130.76$, $129.05,127.34,125.85,122.57,121.06,118.79,118.33$, $118.16,110.09,38.60$.

双(3,3'-1H-吲哚)(4-硝基苯基)甲烷(4d)：粉色固体， $340 \mathrm{mg}$, 产率 93\%. m.p. 217 $219{ }^{\circ} \mathrm{C}$ (lit. ${ }^{[20]} 217 \sim 220$ $\left.{ }^{\circ} \mathrm{C}\right) ; R_{\mathrm{f}}=0.43$ [ $V($ 乙酸乙酯 $): ~ V($ 石 油醚 $\left.)=3: 7\right] .{ }^{1} \mathrm{H}$
NMR (399 MHz, DMSO ) $\delta: 10.94$ (d, $J=1.7 \mathrm{~Hz}, 2 \mathrm{H})$, $8.18 \sim 8.13(\mathrm{~m}, 2 \mathrm{H}), 7.61(\mathrm{~d}, J=8.7 \mathrm{~Hz}, 2 \mathrm{H}), 7.37$ (d, $J=$ $8.1 \mathrm{~Hz}, 2 \mathrm{H}), 7.30(\mathrm{~d}, J=7.9 \mathrm{~Hz}, 2 \mathrm{H}), 7.08 \sim 7.03(\mathrm{~m}, 2 \mathrm{H})$, $6.92 \sim 6.86(\mathrm{~m}, 4 \mathrm{H}), 6.03(\mathrm{~s}, 1 \mathrm{H}) ;{ }^{13} \mathrm{C} \mathrm{NMR}(100 \mathrm{MHz}$, DMSO- $\left.d_{6}\right) \delta: 153.63,146.25,137.09,129.95,126.86$, $124.36,123.91,121.60,119.41,118.92,117.17,112.09$, 39.39 .

双(3,3'-1H-吲哚)(4-甲氧基苯基)甲烷(4e)：粉色固 体, 产率 82\%. m.p. $185 \sim 186{ }^{\circ} \mathrm{C}$ (lit. ${ }^{[20]} 185 \sim 187{ }^{\circ} \mathrm{C}$ ); $R_{\mathrm{f}}=0.59$ [ $V($ 乙酸乙酯 $): V($ 石油醚 $\left.)=3: 7\right] .{ }^{1} \mathrm{H}$ NMR $\left(400 \mathrm{MHz}_{\mathrm{CDCl}}\right) \delta: 7.78(\mathrm{~s}, 2 \mathrm{H}), 7.32(\mathrm{~d}, J=7.9 \mathrm{~Hz}$, $2 \mathrm{H}), 7.27$ (d, $J=8.2 \mathrm{~Hz}, 2 \mathrm{H}), 7.20 \sim 7.14(\mathrm{~m}, 2 \mathrm{H}), 7.12 \sim$ $7.06(\mathrm{~m}, 2 \mathrm{H}), 6.93(\mathrm{dd}, J=11.1,4.0 \mathrm{~Hz}, 2 \mathrm{H}), 6.78 \sim 6.72$ $(\mathrm{m}, 2 \mathrm{H}), 6.59 \sim 6.52(\mathrm{~m}, 2 \mathrm{H}), 5.77(\mathrm{~s}, 1 \mathrm{H}), 3.74 \sim 3.68(\mathrm{~m}$, $3 \mathrm{H}) ;{ }^{13} \mathrm{C} \mathrm{NMR}\left(100 \mathrm{MHz}, \mathrm{CDCl}_{3}\right) \delta: 156.72,135.65$, $135.18,128.55,126.02,122.47,120.84,118.93,118.14$, 112.53, 109.96, 54.17, 38.30.

双(3,3'-1H-吲哚)(3-溴苯基)甲烷(4f)：白色固体，产 率 97\%. m.p. $137 \sim 138{ }^{\circ} \mathrm{C}$ (lit. ${ }^{[20]} 135 \sim 137{ }^{\circ} \mathrm{C}$ ); ${ }^{1} \mathrm{H}$ NMR (400 MHz, $\left.\mathrm{CDCl}_{3}\right) \delta: 7.71(\mathrm{~s}, 2 \mathrm{H}), 7.32(\mathrm{~d}, J=1 \mathrm{~Hz}$, $1 \mathrm{H}), 7.21 \sim 7.15(\mathrm{~m}, 5 \mathrm{H}), 7.11 \sim 7.06(\mathrm{~m}, 1 \mathrm{H}), 6.95 \sim 7.01$ $(\mathrm{m}, 3 \mathrm{H}), 6.87 \sim 6.81(\mathrm{~m}, 2 \mathrm{H}), 6.47 \sim 6.43(\mathrm{~m}, 2 \mathrm{H}), 5.67(\mathrm{~s}$, $1 \mathrm{H}) ;{ }^{13} \mathrm{C} \mathrm{NMR}\left(100 \mathrm{MHz}, \mathrm{CDCl}_{3}\right) \delta: 146.59,136.76$, 131.79 , 129.93, 129.46, 127.47, 126.95, 123.76, 122.49, $122.19,119.84,119.48,119.03,111.22,40.03$.

双(3,3'-1H-吲哚)(3-氨基苯基)甲烷(4g)：白色固体， 产率 50\%. m.p. $187 \sim 189{ }^{\circ} \mathrm{C}$; ${ }^{1} \mathrm{H}$ NMR (400 MHz, DMSO-d $\left.d_{6}\right) \delta: 10.79(\mathrm{~s}, 2 \mathrm{H}), 7.32 \sim 7.37(\mathrm{~m}, 4 \mathrm{H}), 7.03 \sim$ $7.07(\mathrm{~m}, 2 \mathrm{H}), 6.83 \sim 6.95(\mathrm{~m}, 5 \mathrm{H}), 6.41 \sim 6.59(\mathrm{~m}, 2 \mathrm{H})$, $6.39 \sim 6.40(\mathrm{~m}, 1 \mathrm{H}), 5.67(\mathrm{~s}, 1 \mathrm{H}), 4.91(\mathrm{~s}, 2 \mathrm{H}) ;{ }^{13} \mathrm{C} \mathrm{NMR}$ $\left(100 \mathrm{MHz}, \mathrm{DMSO}-d_{6}\right) \delta: 148.34,145.61,136.58,128.45$, $126.8,123.50,120.81,119.23,118.36,118.11,116.34$, $114.14,111.75,111.42,54.95$. HRMS (ESI) calcd for $\mathrm{C}_{23} \mathrm{H}_{19} \mathrm{~N}_{3}(\mathrm{M}+\mathrm{H})^{+}$338.1652, found 338.1656.

1,1-双(3,3'-1H-吲哚)乙烷 $(\mathbf{4 h})$ : 白色固体, 产率 68\%. m.p. $147 \sim 149{ }^{\circ} \mathrm{C}$ (lit. $\left.{ }^{[20]} 148 \sim 150{ }^{\circ} \mathrm{C}\right){ }^{1}{ }^{1} \mathrm{H}$ NMR (400 MHz, DMSO- $d_{6}$ ) $\delta: 10.76(\mathrm{~d}, J=20.9 \mathrm{~Hz}, 2 \mathrm{H}), 7.47$ (d, $J=7.9 \mathrm{~Hz}, 2 \mathrm{H}), 7.35$ (dd, $J=13.7,8.0 \mathrm{~Hz}, 2 \mathrm{H}), 7.15$ $(\mathrm{d}, J=2.1 \mathrm{~Hz}, 2 \mathrm{H}), 7.03(\mathrm{~m}, 2 \mathrm{H}), 6.94 \sim 6.85(\mathrm{~m}, 2 \mathrm{H})$, 4.59 (q, $J=7.0 \mathrm{~Hz}, 1 \mathrm{H}), 1.76(\mathrm{~d}, J=7.1 \mathrm{~Hz}, 3 \mathrm{H}) ;{ }^{13} \mathrm{C}$ NMR (100 MHz, DMSO- $\left.d_{6}\right) \delta$ : 136.58, 126.47, 121.58, 120.61, 120.04, 119.06, 117.86, 111.32, 27.87, 21.90.

2,2-双(3,3'-1H-吲哚)丙烷(4i): 白色固体, 产率 68\%. m.p. $109 \sim 110{ }^{\circ} \mathrm{C}$ (lit. $\left.{ }^{[22]} 108 \sim 110{ }^{\circ} \mathrm{C}\right) ;{ }^{1} \mathrm{H}$ NMR $(400$ MHz, DMSO- $\left.d_{6}\right) \delta: 10.76(\mathrm{~d}, J=20.9 \mathrm{~Hz}, 2 \mathrm{H}), 7.47$ (d, 
$J=7.9 \mathrm{~Hz}, 2 \mathrm{H}), 7.35$ (dd, $J=13.7,8.0 \mathrm{~Hz}, 2 \mathrm{H}), 7.15$ (d, $J=2.1 \mathrm{~Hz}, 2 \mathrm{H}), 6.99 \sim 7.07(\mathrm{~m}, 2 \mathrm{H}), 6.94 \sim 6.85(\mathrm{~m}, 2 \mathrm{H})$, 4.59 (q, $J=7.0 \mathrm{~Hz}, 1 \mathrm{H}), 1.76(\mathrm{~d}, J=7.1 \mathrm{~Hz}, 3 \mathrm{H}) ;{ }^{13} \mathrm{C}$ NMR $\left(100 \mathrm{MHz}, \mathrm{DMSO}-d_{6}\right) \delta: 136.58,126.47,121.58$, 120.61, 120.04, 119.06, 117.86, 111.32, 27.87, 21.90.

3-乙氧基-3-(3'-1H-吲哚)-1-丙炔(5a): 白色固体, 产 率 35\%. m.p. $99 \sim 101{ }^{\circ} \mathrm{C} ;{ }^{1} \mathrm{H}$ NMR $(400 \mathrm{MHz}$, DMSO- $\left.d_{6}\right) \delta: 11.10(\mathrm{~s}, 1 \mathrm{H}), 7.69(\mathrm{~d}, J=7.9 \mathrm{~Hz}, 1 \mathrm{H})$, $7.36 \sim 7.46(\mathrm{~m}, 2 \mathrm{H}), 7.16 \sim 7.09(\mathrm{~m}, 1 \mathrm{H}), 7.06 \sim 7.00(\mathrm{~m}$, $1 \mathrm{H}), 5.50(\mathrm{~d}, J=2.1 \mathrm{~Hz}, 1 \mathrm{H}), 3.66 \sim 3.46(\mathrm{~m}, 2 \mathrm{H}), 1.19 \sim$ $1.07(\mathrm{~m}, 3 \mathrm{H}) ;{ }^{13} \mathrm{C}$ NMR $\left(100 \mathrm{MHz}, \mathrm{DMSO}-d_{6}\right) \delta: 136.55$, $125.58,124.50,121.43,119.23,118.87,112.68,111.64$, $82.65,75.95,64.09,62.18,15.08$. HRMS (ESI) calcd for $\mathrm{C}_{13} \mathrm{H}_{13} \mathrm{NO}(\mathrm{M}+\mathrm{H})^{+} 200.1070$, found 200.1075.

3,3-双(3,3'-1H-吲哚)-1-丙炔(5b): 白色固体，产率 12\%. m.p. $129 \sim 131{ }^{\circ} \mathrm{C} ;{ }^{1} \mathrm{H}$ NMR (400 MHz, DMSO- $d_{6}$ ) $\delta: 11.47(\mathrm{~s}, 2 \mathrm{H}), 8.15(\mathrm{~d}, J=7.9 \mathrm{~Hz}, 2 \mathrm{H}), 7.90 \sim 7.94(\mathrm{~m}$, $4 \mathrm{H}), 7.60 \sim 7.66(\mathrm{~m}, 2 \mathrm{H}), 7.47 \sim 7.55(\mathrm{~m}, 2 \mathrm{H}), 6.12(\mathrm{~d}, J=$ $2.1 \mathrm{~Hz}, 1 \mathrm{H}), 3.76(\mathrm{t}, J=2.3 \mathrm{~Hz}, 1 \mathrm{H}) ;{ }^{13} \mathrm{C} \mathrm{NMR}(100 \mathrm{MHz}$, DMSO- $\left.d_{6}\right) \delta: 136.60,125.72,122.84,120.95,119.10$, $118.20,114.41,111.50,85.57,71.66,25.70$. HRMS (ESI) calcd for $\mathrm{C}_{19} \mathrm{H}_{14} \mathrm{~N}_{2}(\mathrm{M}+\mathrm{H})^{+}$271.1230, found 271.1228.

辅助材料(Supporting Information) 化合物 $3 \mathbf{a} \sim 3 \mathbf{h}$ 和 4a 4i 的氢谱和碳谱以及新化合物的高分辨质谱. 这些 材料可以免费从本刊网站(http://sioc-journal.cn/)上下载.

\section{References}

[1] (a) Shiri, M.; Zolfigol, M. A.; Kruger, H. G.; Tanbakouchian, Z. Chem. Rev. 2010, 110, 2250.

(b) Abe, T.; Nakamura, S.; Yanada, R.; Choshi, T.; Hibino, S.; Ishikura, M. Org. Lett. 2013, 15, 3622.

(c) Pang, Y. Y.; Xu, Z. L. Chin. J. Org. Chem. 2005, 25, 25 (in Chinese).

(庞翼燕, 许遵乐, 有机化学, 2005, 25, 25.)

(d) Pathak, T. P.; Osiak, J. G.; Vaden, R. M.; Welm, B. E.; Sigman, M. S. Tetrahedron 2012, 68, 5203.

(e) Gao, X.; Gong, H. J.; Men, P.; Zhou, L.; Ye, D. Y. Chin. J. Chem. 2013, 31, 1164.

(f) Zhang, J.; Da, Sh. J.; Feng, X. L.; Chen, X. Y. Chin. J. Chem. 2013, 31, 123.

(g) Fang, H. B.; Jin, L.; Huang, N. Y.; Wang, J. Zh.; Zou, K.; Luo, Z. G. Chin. J. Chem. 2013, 31, 831.
[2] Safe, S.; Papineni, S.; Chintharlapalli, S. Cancer Lett. 2008, 269, 326.

[3] (a) Wang, J. R.; Tsai, C. H.; Kulp, S. K.; Chen, C. S. Cancer Lett. 2008, 262, 153 .

(b) Hall, J. M.; Barhoover, M. A.; Kazmin, D.; McDonnell, D. P.; Greenlee, W. F.; Thomas, R. S. Mol. Endocrinol. 2010, 24, 359.

(c) Garikapaty, V. P. S.; Ashok, B. T.; Tadi, K.; Mittelman, A.; Tiwari, R. K. Biochem. Biophys. Res. Commun. 2006, 340, 718.

[4] Bell, R.; Carmeli, S.; Sar, N. J. Nat. Prod. 1994, 57, 1587.

[5] Peng, Y. Y.; Zhang, Q. L.; Yuan, J. J.; Cheng, J. P. Chin. J. Chem. 2008, 26, 2228.

[6] An, L. T.; Ding, F. Q.; Zou, J. P.; Lu, X. H.; Zhang, L. L. Chin. J. Chem. 2007, 25, 822.

[7] He, F.; Li, P.; Gu, Y.; Li, G. Green Chem. 2009, 11, 1767.

[8] Mendes, S. R.; Thurow, S.; Fortes, M. P.; Penteado, F.; Lenardão, E. J.; Alves, D.; Perin, G.; Jacob, R. G. Tetrahedron Lett. 2012, 53, 5402.

[9] Khaksar, S.; Talesh, S. M. J. Fluorine Chem. 2012, 135, 87.

[10] Azizi, N.; Torkian, L.; Saidi, M. R. J. Mol. Catal. A: Chem. 2007, $275,109$.

[11] Zhang, Z.-H.; Yin, L.; Wang, Y.-M. Synthesis 2005, 1949.

[12] Mulla, S. A. R.; Sudalai, A.; Pathan, M. Y.; Siddique, S. A.; Inamdar, S. M.; Chavan, S. S.; Reddy, R. S. RSC $A d v$. 2012, 2, 3525 .

[13] Mo, L. P.; Ma, Z. C.; Zhang, Z. H. Synth. Commun. 2005, 35, 1997.

[14] (a) Ji, S. J.; Zhou, M. F.; Gu, D. G.; Wang, S. Y.; Loh, T. P. Synlett 2003, 2077.

(b) Armstrong, E. L.; Grover, H. K.; Kerr, M. A. J. Org. Chem. 2013, 78, 10534 .

[15] Lin, H.; Zang, Y.; Sun, X.; Lin, G. Chin. J. Chem. 2012, 30, 2309.

[16] Chen, R. E.; Wang, Y. L.; Chen, Z. W.; Su, W. K. Can. J. Chem. 2008, $86,875$.

[17] (a) Fujioka, H.; Minamitsuji, Y.; Moriya, T.; Okamoto, K.; Kubo, O.; Matsushita, T.; Murai, K. Chem. Asian J. 2012, 7, 1925.

(b) Du, T. J.; Wu, Q. P.; Liu, H. X.; Chen, X.; Shu, Y. N.; Xi, X. D.; Zhang, Q. S.; Li, Y. Z. Tetrahedron 2011, 67, 1096.

(c) Huang, L.; Yang, H. B.; Zhang, D. H.; Zhang, Z.; Tang, X. Y.; Xu, Q.; Shi, M. Angew. Chem., Int. Ed. 2013, 52, 6767.

(d) Wu, Q.; Xi, X.; Chen, X.; Li, H.; Zhang, Q.; Li, Y. Chin. J. Chem. 2009, 27, 1962.

[18] (a) Fujioka, H.; Okitsu, T.; Ohnaka, T.; Li, R.; Kubo, O.; Okamoto, K.; Sawama, Y.; Kita, Y. J. Org. Chem. 2007, 72, 7898.

(b) Fujioka, H.; Okitsu, T.; Sawama, Y.; Murata, N.; Li, R.; Kita, Y. J. Am. Chem. Soc. 2006, 128, 5930.

(c) Maegawa, T.; Fujioka, H. J. Synth. Org. Chem. Jpn. 2013, 71, 694.

[19] Chen, R. E.; Wang, Y. L.; Chen, Z. W.; Su, W. K. Can. J. Chem. 2008, $86,875$.

[20] Ganguly, N. C.; Mondal, P.; Barik, S. K. Green Chem. Lett. Rev. 2011, 5, 73.

[21] Yang, Y. L.; Xie, Z. F.; Wang, J. D. Chin. J. Chem. 2011, 29, 2091.

[22] Nadkarni, S. V.; Nagarkar, J. M. Green Chem. Lett. Rev. 2011, 4, 121. 